\title{
The Coupling Characteristic Investigation of Double-Gimbal Magnetically Suspended Control Moment Gyro Used on Agile Maneuver Spacecraft
}

\author{
Peiling Cui, ${ }^{1,2}$ Jian Cui, ${ }^{1,2}$ Qian Yang, ${ }^{1}$ and Shiqiang Zheng ${ }^{1,2}$ \\ ${ }^{1}$ School of Instrumentation Science and Optoelectronics Engineering, Beihang University, Beijing 100191, China \\ ${ }^{2}$ Science and Technology on Inertial Laboratory, Beijing 100191, China \\ Correspondence should be addressed to Peiling Cui; cuiplhh@126.com
}

Received 4 November 2014; Revised 27 January 2015; Accepted 10 February 2015

Academic Editor: Paulo Batista Gonçalves

Copyright (C) 2015 Peiling Cui et al. This is an open access article distributed under the Creative Commons Attribution License, which permits unrestricted use, distribution, and reproduction in any medium, provided the original work is properly cited.

Double-gimbal magnetically suspended CMG is a novel attitude control actuator for the agile maneuver spacecraft. Taking the double-gimbal magnetically suspended control moment gyro used on agile maneuver spacecraft as the research object, the dynamic model of the magnetically suspended rotor, the inner gimbal, and the outer gimbal of double-gimbal magnetically suspended control moment gyro is built. The nonlinear coupling characteristic between the rotor, the gimbal, and the spacecraft is given. It can be seen that the motion of magnetically suspended rotor does not only rely on magnetic bearing force but also suffer from the influence of gimbal servo system and spacecraft motion. The coupling torque includes the gyro coupling torque and the inertial coupling torque. The work in this paper provides the foundation for further studies.

\section{Introduction}

Double-gimbal magnetically suspended control moment gyro (CMG) is a novel solution to realize high precision, long life, and attitude maneuver control of spacecraft by incorporating the advantages of magnetic bearing with double gimbals $[1,2]$. Compared with single-gimbal magnetically suspended $\mathrm{CMG}$, double-gimbal magnetically suspended CMG has more than one degree of freedom, and the singularity is smaller. Therefore, it is believed that doublegimbal magnetically suspended CMG can implement the rapid attitude maneuver with high efficiency $[3,4]$. By the rotation of inner gimbal and outer gimbal, the angular momentum direction of magnetically suspended high-speed rotor is changed, and then the gyro torque is produced. For magnetic bearing, there are five more degrees of freedom than mechanical stator, including three translation motions and two radial rotation motions $[5,6]$.

Double-gimbal magnetically suspended CMG is composed of high-speed magnetically suspended rotor system, inner gimbal servo system, and outer gimbal servo system [7]. In the agile maneuver spacecraft using double-gimbal magnetically suspended CMG as actuator, there is strong coupling torque on the magnetic bearing resulting from the motion of spacecraft and gimbal, and then the high-speed rotor displacement is increased. When this phenomenon is serious, the rotor will collide with the protecting bearing, and the stability of magnetically suspended rotor will be reduced and even lost. Moreover, the spacecraft maneuver and the rotor radial motion will bring disturbance to the gimbal motion. The output torque precision of doublegimbal magnetically suspended control moment gyro will be influenced. Finally, the spacecraft attitude control precision will be reduced when the gimbal motion and rotor radial motion are large. It is important to build the dynamic model of magnetically suspended rotor, inner gimbal, outer gimbal, and spacecraft when using double-gimbal magnetically suspended control moment gyro as the spacecraft attitude control actuator.

For spacecraft dynamic modeling based on mechanical CMG, there is some research result. In [8], the mechanical CMG model is given, and the vector-form output torque of CMG in spacecraft body frame is provided. In [9], the dynamic model of one mechanical double-gimbal CMG is 


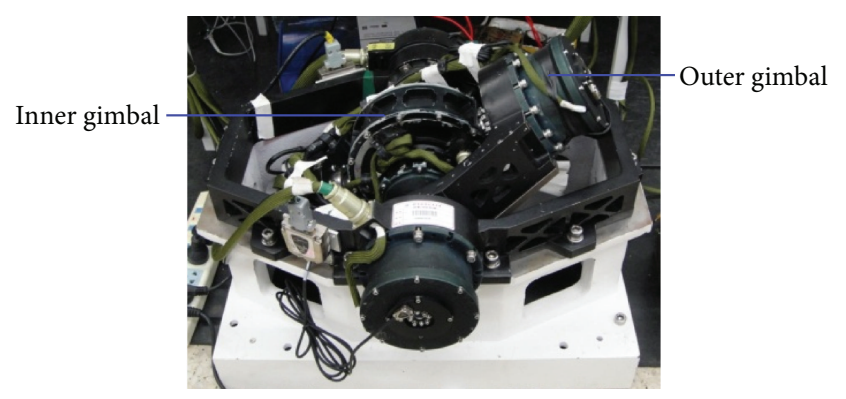

FIGURE 1: Prototype of double-gimbal magnetically suspended CMG.

built using Lagrange equation, and the adaptive feedback control law is put forward. In [10], the inertial torque of inner gimbal and outer gimbal is considered, and the spacecraft attitude dynamic model with a cluster of double-gimbal CMGs is built. For spacecraft dynamic modeling based on magnetically suspended CMG, there is little research result. In [11], the spacecraft dynamic model using singlegimbal magnetically suspended CMG is built. It is aimed at large spacecraft, the spacecraft angular velocity is limited to $10^{-2} \mathrm{rad} / \mathrm{s}$, and the effect of spacecraft maneuver is not involved. As for double-gimbal magnetically suspended CMG, investigation mainly focuses on single part of the magnetically suspended CMG, such as rotor system $[12,13]$ and gimbal servo system [14]. Models are built separately, omitting the coupling characteristic between different parts. Besides, researchers do not consider maneuver of the spacecraft.

In this paper, taking double-gimbal magnetically suspended CMG used for attitude control of agile maneuver spacecraft as the research object, the dynamic model of magnetically suspended rotor, inner gimbal, and outer gimbal of double-gimbal magnetically suspended CMG is built. The nonlinear coupling characteristic between rotor, gimbal, and spacecraft is investigated.

\section{The Working Principal of Double-Gimbal Magnetically Suspended CMG}

Double-gimbal magnetically suspended CMG consists of three subsystems, including magnetically suspended highspeed rotor system, inner gimbal rate servo system, and outer gimbal rate servo system. The angular momentum is provided by magnetically suspended high-speed rotor. The torque is output by the direction changing of angular momentum, which will adjust the spacecraft attitude.

Double-gimbal magnetically suspended CMG prototype is shown in Figure 1. The gyro room and its subsidiary control system is called the magnetically suspended high-speed rotor system, including the magnetic bearing system and the motor for the high-speed rotor. The gyro room is equipped with the components, including the motor of rotor, axial magnetic bearing, radial magnetic bearing, the displacement sensor, and the protecting bearing. The rotor is driven by the motor, which will provide constant angle momentum. The rotor is suspended by the magnetic bearing, and it has five degrees of freedom. This kind of magnetic bearing is called fivedegree-of-freedom magnetic bearing. The displacement of each degree of freedom is measured by the displacement sensor. If the rotor is not on the given zero position, the error signal is passed through the magnetic bearing controller. The amplifier will output the corresponding control current, which will drive the magnetic bearing to produce bearing force to return the rotor to the given position. The base is the mechanical structure that connects double-gimbal magnetically suspended CMG with spacecraft. The output torque of double-gimbal magnetically suspended CMG is imparted to spacecraft by the base.

The magnetic bearing is an elasticity support with gap. When it is suspended normally, the magnetically suspended rotor has small rotation angle and translation motion relative to gyro room. Meanwhile, the angle motion of magnetically suspended high-speed rotor is interrelated with the rotation of inner gimbal and outer gimbal. The reference frames should be defined firstly.

At the initial time, it is defined that the gimbal angle, the gimbal rate, the base angle position, and the base angular velocity are all zero. For spacecraft dynamic modeling, the coordinate frames are defined, which are illustrated in Figure 2.

$O_{i} X_{i} Y_{i} Z_{i}$ is the inertial frame. The origin is earth center of mass. $O_{i} X_{i}$ points to vernal equinox; $O_{i} Z_{i}$ points to the north pole.

$O_{o} X_{o} Y_{o} Z_{o}$ is the orbit frame. The origin is the spacecraft center of mass. $O_{o} X_{o}$ and $O_{o} Y_{o}$ represent the roll axis and the pitch axis, respectively. Both of them are located in the orbit plane. The roll axis points to the go-ahead direction. The pitch axis is vertical to orbit plane. $O z_{o}$ represents the yaw axis, which points to earth's core. This frame rotates with speed $\omega_{o}$, and the rotation direction is contrary to the pitch axis.

$\mathrm{O}_{b} x_{b} y_{b} z_{b}$ is the spacecraft body frame. The origin is the spacecraft center of mass, $O x_{b}, O y_{b}$, and $O z_{b}$ are fixed on the spacecraft, and they are the moments of inertial principal axis of the spacecraft. $\left[\begin{array}{lll}\varphi & \theta & \psi\end{array}\right]^{T}$ is the 3-1-2 Euler angle with respect to orbit frame.

$\mathrm{O}_{s} x_{s} y_{s} z_{s}$ is the zero position frame. At the zero position, it coincides with the outer gimbal frame. It is fixed with body frame.

$O_{j} x_{j} y_{j} z_{j}$ is the outer gimbal frame. It is fixed with outer gimbal. The outer gimbal angle is $\theta_{j}$. It is positive when the rotor rotation speed $\omega_{j}$ along $y_{j}$ direction is positive.

$\mathrm{O}_{g} x_{g} y_{g} z_{g}$ is the inner gimbal frame. It is fixed with inner gimbal. The inner gimbal angle is $\theta_{g}$. It is positive when the rotor rotation speed $\omega_{g}$ along $x_{g}$ direction is positive.

$O_{1} x_{1} y_{1} z_{1}$ is the stator frame. It is fixed with gyro room. This frame keeps still with respect to inner gimbal frame. $O x_{1}$ and $O y_{1}$ point to central directions of the radial magnetic bearing.

$\mathrm{O}_{2} x_{2} y_{2} z_{2}$ is the inner loop frame. It is fixed with the suppositional inner loop. This frame has three translation motions and two rotation degrees of freedom. $\mathrm{O}_{2}$ is the center of rotor mass. $x_{g 1} y_{g 1} z_{g 1}$ and $x_{g 2} y_{g 2} z_{g 2}$ in Figure 3 are the suppositional inner loop and the dummy outer loop frames, respectively. The outer gimbal angle $\alpha$ is the Euler angle of 

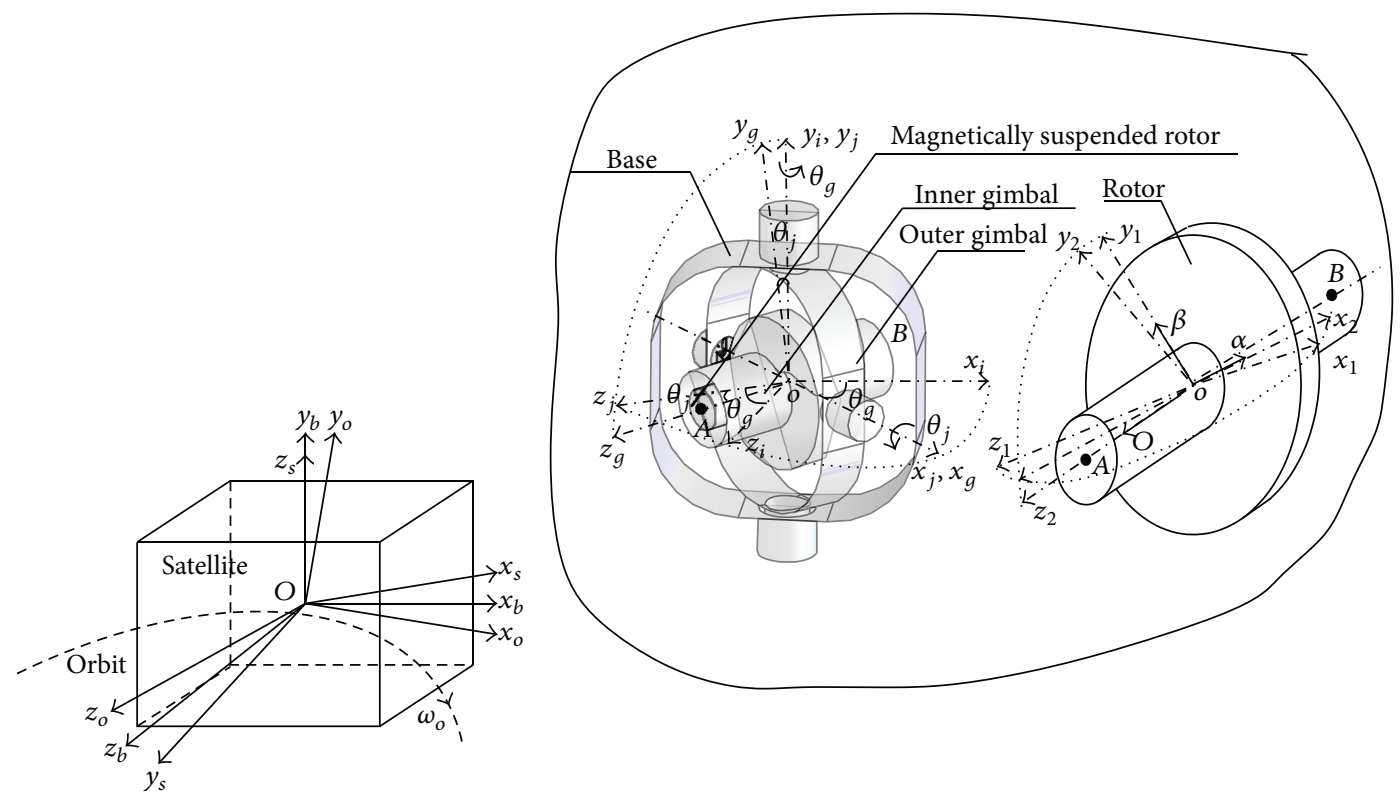

Figure 2: The illustration of coordinate frames.

$x_{g 1} y_{g 1} z_{g 1}$ with respect to $O x_{1} y_{1} z_{1}$. The inter gimbal angle $\beta$ is the Euler angle of $x_{g 2} y_{g 2} z_{g 2}$ with respect to $x_{g 1} y_{g 1} z_{q 1}$.

$\mathrm{O}_{r} x_{r} y_{r} z_{r}$ is the rotor frame. This frame is fixed with rotor and has one rotation degree of freedom along $z_{r}$ axis with respect to inner loop frame. The rotor rotation angle is $\Omega t$. $\Omega$ is the constant angular velocity, and it is expressed in rotor frame coordinate as $[0,0, \Omega]^{T}$.

$\mathrm{C}_{a}^{b}$ is used to denote the coordinate transformation matrix from frame $a$ to frame $b . \mathbf{y}_{/ m}$ denotes the vector projected in coordinate $m . \mathbf{x}^{\times}$denotes the skew symmetric matrix of $\mathbf{x}=\left[\begin{array}{lll}x_{1} & x_{2} & x_{3}\end{array}\right]^{T}$. On the condition of small angle transformation, then

$$
\begin{aligned}
& \mathbf{C}_{o}^{b}=\left[\begin{array}{ccc}
\cos \theta & 0 & -\sin \theta \cos \phi \\
0 & 1 & \sin \phi \\
\sin \theta & 0 & \cos \theta \cos \phi
\end{array}\right] \approx\left[\begin{array}{ccc}
1 & \psi & -\theta \\
-\psi & 1 & \varphi \\
\theta & -\varphi & 1
\end{array}\right], \\
& \mathbf{C}_{b}^{s}=\left[\begin{array}{lll}
1 & 0 & 0 \\
0 & 0 & 1 \\
0 & 1 & 0
\end{array}\right], \quad \mathbf{C}_{s}^{j}=\left[\begin{array}{ccc}
\cos \theta_{j} & 0 & -\sin \theta_{j} \\
0 & 1 & 0 \\
\sin \theta_{j} & 0 & \cos \theta_{j}
\end{array}\right] \text {, } \\
& \mathbf{C}_{j}^{g}=\left[\begin{array}{ccc}
1 & 0 & 0 \\
0 & \cos \theta_{g} & \sin \theta_{g} \\
0 & -\sin \theta_{g} & \cos \theta_{g}
\end{array}\right], \quad \mathbf{C}_{g}^{1}=\left[\begin{array}{lll}
1 & 0 & 0 \\
0 & 1 & 0 \\
0 & 0 & 1
\end{array}\right], \\
& \mathbf{C}_{1}^{g 1}=\left[\begin{array}{ccc}
1 & 0 & 0 \\
0 & \cos \alpha & \sin \alpha \\
0 & -\sin \alpha & \cos \alpha
\end{array}\right] \\
& \mathbf{C}_{g 1}^{g 2}=\left[\begin{array}{ccc}
\cos \beta & 0 & -\sin \beta \\
0 & 1 & 0 \\
\sin \beta & 0 & \cos \beta
\end{array}\right] \text {, }
\end{aligned}
$$

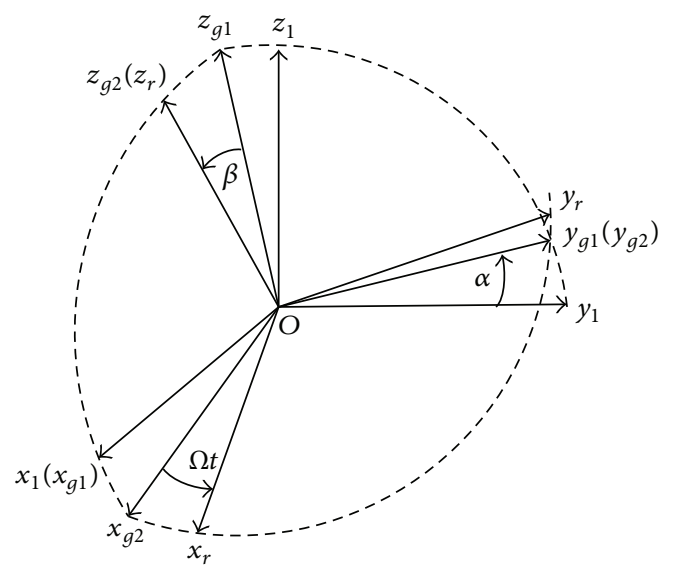

FIGURE 3: The coordinate transformation matrix from the stator frame to the rotor frame.

$$
\begin{aligned}
& \mathbf{C}_{1}^{2}=\mathbf{C}_{1}^{g 1} \mathbf{C}_{g 1}^{g 2} \approx\left[\begin{array}{ccc}
1 & 0 & -\beta \\
0 & 1 & \alpha \\
\beta & -\alpha & 1
\end{array}\right], \\
& \mathbf{C}_{2}^{r}=\left[\begin{array}{ccc}
\cos \Omega t & \sin \Omega t & 0 \\
-\sin \Omega t & \cos \Omega t & 0 \\
0 & 0 & 1
\end{array}\right] .
\end{aligned}
$$

\section{The Dynamic Model of Double-Gimbal Magnetically Suspended CMG}

3.1. The Dynamic Model of Magnetically Suspended Rotor. In order to obtain large rotor angular momentum, the rotor is 

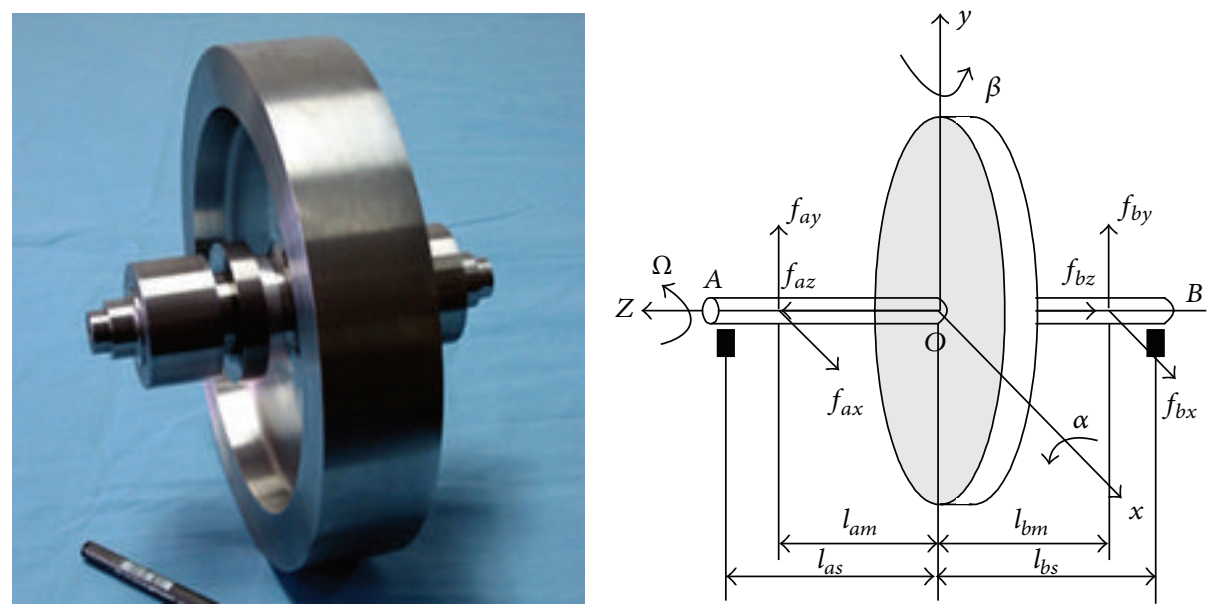

FIGURE 4: The sketch map of magnetic bearing dynamic model.

designed as disc shape to improve the moment of inertia. The moment of inertia ratio between the pole and the equator is large. In order to perform the dynamic analysis and control system design, the magnetic bearing dynamic model of magnetically suspended CMG must be built. The main nomenclature used in this paper is listed in the Nomenclature section.

The magnetically suspended rotor has six degrees of freedom, including translation motion along $x, y$, and $z$ direction and rotation motion along $x, y$, and $z$ direction. The rotation along $z$ direction is controlled by the motor. The other five degrees of freedom are controlled by magnetic bearing, including the translation motion along $x, y$, and $z$ direction and the radial rotation along $x, y$ direction. The exclusive force between the magnetically suspended rotor and the gyro room is the magnetic bearing force. In Figure 4, the radial bearing forces $f_{a x}$ and $f_{b x}$ can be synthesized into radial force $f_{x}$. The radial bearing forces $f_{a y}$ and $f_{b y}$ can be synthesized into radial force $f_{y}$. The radial torque in $x$ and $y$ direction is $p_{y}$ and $p_{x}$, which will influence the radial translation and the rotation of magnetically suspended rotor. When the magnetic bearing rotates about the centroid, the translation and rotation motion about the rotor centroid can be considered separately. Firstly, the translation and rotation motion equations of the rotor must be built. By synthesizing the equations, the dynamic equation of the magnetically suspended rotor can be obtained.

The relative motion of magnetically suspended rotor in noninertial frame suffers from two kinds of force. One is the active control force of magnetic bearing and the other is the implicated inertial force $m \overrightarrow{\mathbf{a}}_{e}$ and Coriolis inertial force $m \overrightarrow{\mathbf{a}}_{c}$ that result from the implicated motion of inner gimbal and outer gimbal. The rotor dynamic equation in noninertial frame is $m \overrightarrow{\mathbf{a}}_{r}=\mathbf{F}+\left(-m \overrightarrow{\mathbf{a}}_{e}\right)+\left(-m \overrightarrow{\mathbf{a}}_{c}\right) \cdot m$ is the mass of magnetically suspended rotor. $\overrightarrow{\mathbf{a}}_{r}$ is the relative translation acceleration of magnetically suspended rotor. $\mathbf{F}$ is the active force, namely, the bearing force. $\overrightarrow{\mathbf{a}}_{e}$ and $\overrightarrow{\mathbf{a}}_{c}$ are the implicated acceleration and Coriolis acceleration. The relative translation acceleration is greater than the implicated acceleration and Coriolis acceleration, so the implicated inertial force and Coriolis inertial force can be ignored. The relative translation motion model of the magnetically suspended rotor is simplified as $m \overrightarrow{\mathbf{a}}_{r}=\mathbf{F}$, where $\overrightarrow{\mathbf{a}}_{r}=$ $\left[\begin{array}{lll}\ddot{x}_{r} & \ddot{y}_{r} & \ddot{z}_{r}\end{array}\right]^{T}, \mathbf{F}=\left[\begin{array}{lll}f_{x} & f_{y} & f_{z}\end{array}\right]^{T}$.

Similar with the translation motion of magnetically suspended rotor, the rotation of magnetically suspended rotor in inertial frame suffers from two kinds of torque. One is the active control torque of magnetic bearing, and the other is implicated inertial torque and Coriolis inertial torque that are produced from the implicated motion of the noninertial frame. Considering the ground experimental condition, the absolute angle motion of magnetically suspended rotor includes the following three kinds of motion.

(1) The rotation of rotor frame relative to magnetic bearing stator is described with Euler angles $\alpha, \beta$, and $\gamma$, which rotates about the inner loop rotation axis, the outer loop rotation axis, and the rotor spin axis.

(2) The rotation of the magnetic bearing stator frame relative to inner gimbal frame is denoted by $\theta_{g}$.

(3) The rotation of outer gimbal frame relative to inertial frame is denoted by $\theta_{j}$.

In the following, based on Newton-Euler method, Euler dynamic equation is used to derive the dynamic model of magnetically suspended rotor of double-gimbal magnetically suspended CMG. The reference frame is the magnetic bearing stator frame $O x_{1} y_{1} z_{1}$, and the movable frame is the inner loop frame $\mathrm{O} x_{2} y_{2} z_{2}$.

The angle speed vector of magnetically suspended rotor $\overrightarrow{\boldsymbol{\omega}}_{i r}$ includes six parts, namely, the rotation speed of rotor frame relative to stator frame $\vec{\omega}_{1 r}$, the rotation speed of stator frame relative to inner gimbal frame $\overrightarrow{\boldsymbol{\omega}}_{g 1}$, the rotation speed of inner gimbal frame relative to outer gimbal frame $\overrightarrow{\boldsymbol{\omega}}_{j g}$, the rotation speed of outer gimbal frame relative to zero position frame $\overrightarrow{\boldsymbol{\omega}}_{s j}$, the rotation speed of zero position frame relative to body frame $\overrightarrow{\boldsymbol{\omega}}_{b s}$, and the rotation speed of body frame relative 
to inertial frame $\vec{\omega}_{i b}: \overrightarrow{\boldsymbol{\omega}}_{i r}=\overrightarrow{\boldsymbol{\omega}}_{i b}+\overrightarrow{\boldsymbol{\omega}}_{b s}+\overrightarrow{\boldsymbol{\omega}}_{s j}+\overrightarrow{\boldsymbol{\omega}}_{j g}+\overrightarrow{\boldsymbol{\omega}}_{g 1}+$ $\vec{\omega}_{1 r}$. Because the projection gap is small, the maximal radial rotation angle of the Euler angles $\alpha, \beta$ is less than $3 \times 10^{-3} \mathrm{rad}$. It can be considered that $\cos \alpha \approx 1, \cos \beta \approx 1, \sin \alpha \approx 0$, $\sin \beta \approx 0$.

(1) Derive the angular velocity projection of the movable frame about each axis. The angular velocity of rotor frame relative to stator frame is $\overrightarrow{\boldsymbol{\omega}}_{1 r}=\dot{\overrightarrow{\boldsymbol{\alpha}}}+\dot{\overrightarrow{\boldsymbol{\beta}}}+\dot{\vec{\gamma}}$. Its projection in inner loop frame is

$$
\overrightarrow{\boldsymbol{\omega}}_{1 r}^{2}=\left[\begin{array}{c}
\dot{\alpha} \\
0 \\
0
\end{array}\right]+\mathbf{C}_{1}^{g 1}\left[\begin{array}{c}
0 \\
\dot{\beta} \\
0
\end{array}\right]+\left[\begin{array}{l}
0 \\
0 \\
\dot{\gamma}
\end{array}\right]=\left[\begin{array}{c}
\dot{\alpha} \\
\dot{\beta} \cos \alpha \\
\dot{\gamma}-\dot{\beta} \sin \alpha
\end{array}\right] .
$$

Because stator frame is still relative to inner gimbal frame, $\vec{\omega}_{g 1}=0$. The angular velocity of inner gimbal relative to outer gimbal is $\overrightarrow{\boldsymbol{\omega}}_{j g}$. Its projection in inner loop frame is

$$
\overrightarrow{\boldsymbol{\omega}}_{j g}^{2}=\mathbf{C}_{1}^{2} \mathbf{C}_{g}^{1} \overrightarrow{\boldsymbol{\omega}}_{j g} \approx\left[\begin{array}{c}
\dot{\theta}_{g} \\
0 \\
0
\end{array}\right] .
$$

The angular velocity of outer gimbal relative to zero position frame is $\overrightarrow{\boldsymbol{\omega}}_{s j}$. Its projection in inner loop frame is

$$
\overrightarrow{\boldsymbol{\omega}}_{s j}^{2}=\mathbf{C}_{1}^{2} \mathbf{C}_{g}^{1} \mathbf{C}_{j}^{g} \overrightarrow{\boldsymbol{\omega}}_{j g}=\left[\begin{array}{c}
0 \\
\dot{\theta}_{j} \cos \theta_{g} \\
-\dot{\theta}_{j} \sin \theta_{g}
\end{array}\right] .
$$

The zero position frame is still relative to body frame, $\overrightarrow{\boldsymbol{\omega}}_{b s}=0$. The rotation speed of spacecraft relative to orbit frame is $(\dot{\varphi}, \dot{\theta}, \dot{\psi})$, which corresponds to roll, pitch, and yaw. The rotation speed of orbit frame is $\left(0,-\omega_{0}, 0\right)^{T}$. So, the rotation speed of spacecraft can be described in frame $O x_{b} y_{b} z_{b}$ as follows:

$$
\begin{aligned}
\overrightarrow{\boldsymbol{\omega}}_{i b}^{b} & =\left[\begin{array}{c}
\omega_{i b x} \\
\omega_{i b y} \\
\omega_{i b z}
\end{array}\right] \\
& =\left[\begin{array}{c}
\dot{\varphi} \\
\dot{\theta} \\
\dot{\psi}
\end{array}\right]+\left[\begin{array}{ccc}
1 & \psi & -\theta \\
-\psi & 1 & \varphi \\
\theta & \varphi & 1
\end{array}\right]\left[\begin{array}{c}
0 \\
-\omega_{0} \\
0
\end{array}\right]=\left[\begin{array}{c}
\dot{\varphi}-\omega_{0} \psi \\
\dot{\theta}-\omega_{0} \\
\dot{\psi}+\omega_{0} \varphi
\end{array}\right] .
\end{aligned}
$$

The spacecraft rotation speed $\overrightarrow{\boldsymbol{\omega}}_{i b}$ in inner loop frame is

$$
\begin{aligned}
\overrightarrow{\boldsymbol{\omega}}_{i b}^{2} & =\left[\begin{array}{l}
\omega_{i b x}^{2} \\
\omega_{i b y}^{2} \\
\omega_{i b z}^{2}
\end{array}\right]=\mathbf{C}_{1}^{2} \mathbf{C}_{g}^{1} \mathbf{C}_{j}^{g} \mathbf{C}_{s}^{j} \mathbf{C}_{b}^{s} \overrightarrow{\boldsymbol{\omega}}_{i b}^{b} \\
= & {\left[\begin{array}{c}
\cos \theta_{j} \omega_{i b x}^{b}-\sin \theta_{j} \omega_{i b y}^{b} \\
\sin \theta_{g} \sin \theta_{j} \omega_{i b x}^{b}+\cos \theta_{g} \omega_{i b z}^{b}+\sin \theta_{g} \cos \theta_{j} \omega_{i b y}^{b} \\
\cos \theta_{g} \sin \theta_{j} \omega_{i b x}^{b}-\sin \theta_{g} \omega_{i b z}^{b}+\cos \theta_{g} \cos \theta_{j} \omega_{i b y}^{b}
\end{array}\right] . }
\end{aligned}
$$

The absolute angular velocity $\overrightarrow{\boldsymbol{\omega}}_{i r}$ of magnetically suspended rotor in inner loop frame is denoted by

$$
\begin{aligned}
\overrightarrow{\boldsymbol{\omega}}_{i r}^{2} & =\mathbf{C}_{b}^{2} \overrightarrow{\boldsymbol{\omega}}_{i b}+\mathbf{C}_{j}^{2} \overrightarrow{\boldsymbol{\omega}}_{s j}+\mathbf{C}_{g}^{2} \overrightarrow{\boldsymbol{\omega}}_{j g}+\overrightarrow{\boldsymbol{\omega}}_{1 r} \\
& =\overrightarrow{\boldsymbol{\omega}}_{i b}^{2}+\overrightarrow{\boldsymbol{\omega}}_{s j}^{2}+\overrightarrow{\boldsymbol{\omega}}_{j g}^{2}+\overrightarrow{\boldsymbol{\omega}}_{1 r}^{2} .
\end{aligned}
$$

Then

$$
\begin{aligned}
\overrightarrow{\boldsymbol{\omega}}_{i r}^{2} & =\left[\begin{array}{c}
\omega_{i b x}^{2}+\dot{\alpha}+\dot{\theta}_{g} \\
\omega_{i b y}^{2}+\dot{\beta} \cos \alpha+\dot{\theta}_{j} \cos \theta_{g} \\
\omega_{i b z}^{2}+\dot{\gamma}-\dot{\beta} \sin \alpha-\dot{\theta}_{j} \sin \theta_{g}
\end{array}\right] \\
= & {\left[\begin{array}{c}
\cos \theta_{j} \omega_{i b x}^{b}-\sin \theta_{j} \omega_{i b y}^{b}+\dot{\alpha}+\dot{\theta}_{g} \\
\sin \theta_{g} \sin \theta_{j} \omega_{i b x}^{b}+\cos \theta_{g} \omega_{i b z}^{b}+\sin \theta_{g} \cos \theta_{j} \omega_{i b y}^{b} \\
+\dot{\beta} \cos \alpha+\dot{\theta}_{j} \cos \theta_{g} \\
\cos \theta_{g} \sin \theta_{j} \omega_{i b x}^{b}-\sin \theta_{g} \omega_{i b z}^{b}+\cos \theta_{g} \cos \theta_{j} \omega_{i b y}^{b} \\
+\dot{\gamma}-\dot{\beta} \sin \alpha-\dot{\theta}_{j} \sin \theta_{g}
\end{array}\right] . }
\end{aligned}
$$

(2) The moment of inertia of magnetic bearing in $o x_{r} y_{r} z_{r}$ is $\mathbf{J}_{r}=\operatorname{diag}\left(\begin{array}{lll}J_{r x} & J_{r y} & J_{r z}\end{array}\right)$. Because the rotor is symmetric, its equator moment of inertia is $J_{r x}=J_{r y}=J_{r r}$, and its pole moment of inertia is $J_{r z}=J_{p}$. So the rotor moment of inertia relative to inner loop frame has the following relationship with $\mathbf{J}_{r}$ :

$$
\mathbf{J}_{2}=\left[\mathbf{C}_{2}^{r}\right]^{T} \mathbf{J}_{r} \mathbf{C}_{2}^{r} \approx\left[\begin{array}{ccc}
J_{r r} & 0 & 0 \\
0 & J_{r r} & 0 \\
0 & 0 & J_{r z}
\end{array}\right]
$$

The rotor angular momentum in inner loop frame is

$$
\begin{aligned}
& \mathbf{H}_{r}=\mathbf{J}_{r} \cdot \overrightarrow{\boldsymbol{\omega}}_{i r}^{2} \\
& =\mathbf{J}_{r} \cdot\left(\mathbf{C}_{b}^{2} \overrightarrow{\boldsymbol{\omega}}_{i b}+\mathbf{C}_{j}^{2} \overrightarrow{\boldsymbol{\omega}}_{s j}+\mathbf{C}_{g}^{2} \overrightarrow{\boldsymbol{\omega}}_{j g}+\overrightarrow{\boldsymbol{\omega}}_{1 r}\right) \\
& =\left[\begin{array}{c}
J_{r r}\left(\omega_{i b x}^{2}+\dot{\alpha}+\dot{\theta}_{g}\right) \\
J_{r r}\left(\omega_{i b y}^{2}+\dot{\beta} \cos \alpha+\dot{\theta}_{j} \cos \theta_{g}\right) \\
J_{r z}\left(\omega_{i b z}^{2}+\dot{\gamma}-\dot{\beta} \sin \alpha-\dot{\theta}_{j} \sin \theta_{g}\right)
\end{array}\right] .
\end{aligned}
$$

Then

$$
\begin{aligned}
\dot{\mathbf{H}}_{r}= & \mathbf{J}_{r} \cdot \dot{\overrightarrow{\boldsymbol{\omega}}}_{i r}^{2} \\
= & \mathbf{J}_{r}\left\{\left(-\overrightarrow{\boldsymbol{\omega}}_{1 r}^{\times} \mathbf{C}_{b}^{2}-\mathbf{C}_{g}^{2} \overrightarrow{\boldsymbol{\omega}}_{j g}^{\times} \mathbf{C}_{b}^{g}-\mathbf{C}_{j}^{2} \overrightarrow{\boldsymbol{\omega}}_{s j}^{\times} \mathbf{C}_{b}^{j}\right) \overrightarrow{\boldsymbol{\omega}}_{i b}+\mathbf{C}_{b}^{2 \dot{\vec{\omega}}_{i b}}\right. \\
& +\left(-\overrightarrow{\boldsymbol{\omega}}_{1 r}^{\times} \mathbf{C}_{j}^{2}-\mathbf{C}_{g}^{2} \overrightarrow{\boldsymbol{\omega}}_{s j}^{\times} \mathbf{C}_{j}^{g}\right) \overrightarrow{\boldsymbol{\omega}}_{s j}+\mathbf{C}_{j}^{2} \dot{\overrightarrow{\boldsymbol{\omega}}}_{s j}
\end{aligned}
$$




$$
\begin{aligned}
& \left.+\left(-\vec{\omega}_{1 r}^{\times} \mathbf{C}_{g}^{2} \vec{\omega}_{j g}\right)+\mathbf{C}_{g}^{2 \dot{\omega}_{j g}}+\dot{\vec{\omega}}_{1 r}\right\} \\
& =\left[\begin{array}{c}
J_{r r}\left(\dot{\omega}_{i b x}^{2}+\ddot{\alpha}+\ddot{\theta}_{g}\right) \\
J_{r r}\left(\dot{\omega}_{i b y}^{2}+\ddot{\beta} \cos \alpha-\dot{\beta} \dot{\alpha} \sin \alpha\right. \\
\left.+\ddot{\theta}_{j} \cos \theta_{g}-\dot{\theta}_{j} \dot{\theta}_{g} \sin \theta_{g}\right) \\
J_{r z}\left(\dot{\omega}_{i b z}^{2}+\ddot{\gamma}-\ddot{\beta} \sin \alpha-\dot{\beta} \dot{\alpha} \cos \alpha\right. \\
\left.-\ddot{\theta}_{j} \sin \theta_{g}-\dot{\theta}_{j} \dot{\theta}_{g} \cos \theta_{g}\right)
\end{array}\right] .
\end{aligned}
$$

$$
\begin{aligned}
J_{r z} & {\left[\dot{\omega}_{i b z}^{2}+\ddot{\gamma}-\ddot{\beta} \sin \alpha-\dot{\beta} \dot{\alpha} \cos \alpha-\ddot{\theta}_{j} \sin \theta_{g}-\dot{\theta}_{j} \dot{\theta}_{g} \cos \theta_{g}\right] } \\
& +J_{r r}\left[\omega_{i b x}^{2}+\dot{\alpha}+\dot{\theta}_{g}\right]\left[\omega_{i b y}^{2}+\dot{\beta} \cos \alpha+\dot{\theta}_{j} \cos \theta_{g}\right] \\
& -J_{r r}\left[\omega_{i b x}^{2}+\dot{\alpha}+\dot{\theta}_{g}\right]\left[\omega_{i b y}^{2}+\dot{\beta} \cos \alpha+\dot{\theta}_{j} \cos \theta_{g}\right]=0 .
\end{aligned}
$$

Because the projection gap is small and the permitted maximal radial rotation angle is less than $3^{\prime \prime}$, the rotor motion

The projection of the angular velocity in inner loop frame can be simplified as

$$
\overrightarrow{\boldsymbol{\omega}}_{i 2}^{2}=\left[\begin{array}{c}
\omega_{i b x}^{2}+\dot{\alpha}+\dot{\theta}_{g} \\
\omega_{i b y}^{2}+\dot{\beta} \cos \alpha+\dot{\theta}_{j} \cos \theta_{g} \\
\omega_{i b z}^{2}-\dot{\beta} \sin \alpha-\dot{\theta}_{j} \sin \theta_{g}
\end{array}\right] .
$$

(3) In this paper, five-degree-of-freedom magnetic bearing is used. The axial magnetic bearing only provides translation force, and it cannot output torque to the rotor. Therefore, the total torque of the rotor is

$$
\mathbf{M}_{r 1}^{2}=\left[\begin{array}{c}
p_{x} \\
p_{y} \\
0
\end{array}\right]
$$

(4) In magnetic bearing frame $O x_{r} y_{r} z_{r}$, the dynamic equation of the $i$ th double-gimbal magnetically suspended CMG is

$$
\begin{aligned}
& \dot{H}_{r x}+H_{r z} \omega_{i 2 y}^{2}-H_{r y} \omega_{i 2 z}^{2}=M_{r 1 x}^{2}, \\
& \dot{H}_{r y}+H_{r x} \omega_{i 2 z}^{2}-H_{r z} \omega_{i 2 x}^{2}=M_{r 1 y}^{2}, \\
& \dot{H}_{r z}+H_{r y} \omega_{i 2 x}^{2}-H_{r x} \omega_{i 2 y}^{2}=M_{r 1 z}^{2},
\end{aligned}
$$

where $M_{r 1 x}^{2}, M_{r 1 y}^{2}$, and $M_{r 1 z}^{2}$ are the elements of $\mathbf{M}_{r 1}^{2}$. According to (2) (14), the rotor dynamic equation is

$$
\begin{aligned}
& J_{r r}\left[\dot{\omega}_{i b x}^{2}+\ddot{\alpha}+\ddot{\theta}_{g}\right] \\
& \quad+J_{r z}\left[\dot{\omega}_{i b z}^{2}+\ddot{\gamma}-\ddot{\beta} \sin \alpha-\dot{\beta} \dot{\alpha} \cos \alpha\right. \\
& \left.\quad-\ddot{\theta}_{j} \sin \theta_{g}-\dot{\theta}_{j} \dot{\theta}_{g} \cos \theta_{g}\right] \\
& \quad \cdot\left[\omega_{i b y}^{2}+\dot{\beta} \cos \alpha+\dot{\theta}_{j} \cos \theta_{g}\right] \\
& \quad-J_{r r}\left[\omega_{i b y}^{2}+\dot{\beta} \cos \alpha+\dot{\theta}_{j} \cos \theta_{g}\right] \\
& \quad \cdot\left[\dot{\omega}_{i b z}^{2}-\dot{\beta} \sin \alpha-\dot{\theta}_{j} \sin \theta_{g}\right]=p_{x}, \\
& J_{r r}\left[\dot{\omega}_{i b y}^{2}+\ddot{\beta} \cos \alpha-\dot{\beta}_{\dot{\alpha}} \sin \alpha+\ddot{\theta}_{j} \cos \theta_{g}-\dot{\theta}_{j} \dot{\theta}_{g} \sin \theta_{g}\right] \\
& \quad+J_{r r}\left[\omega_{i b x}^{2}+\dot{\alpha}+\dot{\theta}_{g}\right]\left[\dot{\omega}_{i b z}^{2}-\dot{\beta}_{\operatorname{\beta in}} \sin -\dot{\theta}_{j} \sin \theta_{g}\right] \\
& \quad-J_{r z}\left[\dot{\omega}_{i b z}^{2}+\dot{\gamma}-\dot{\beta}_{\operatorname{\beta in}} \sin -\dot{\theta}_{j} \sin \theta_{g}\right]\left[\omega_{i b x}^{2}+\dot{\alpha}+\dot{\theta}_{g}\right]=p_{y},
\end{aligned}
$$

is restricted in the projection gap. $\alpha$ and $\beta$ are small, $\dot{\gamma} \gg \dot{\alpha}$, $\dot{\gamma} \gg \dot{\beta}, J_{r r}<J_{r z}$, so the above equation can be simplified as

$$
\begin{gathered}
J_{r r}\left(\dot{\omega}_{i b x}^{2}+\ddot{\alpha}+\ddot{\theta}_{g}\right)+H_{r z}\left(\omega_{i b y}^{2}+\dot{\beta}+\dot{\theta}_{j} \cos \theta_{g}\right)=p_{x}, \\
J_{r r}\left(\dot{\omega}_{i b y}^{2}+\ddot{\beta}+\ddot{\theta}_{j} \cos \theta_{g}-\dot{\theta}_{j} \dot{\theta}_{g} \sin \theta_{g}\right) \\
-H_{r z}\left(\omega_{i b x}^{2}+\dot{\alpha}+\dot{\theta}_{g}\right)=p_{y}, \\
J_{r z}\left(\dot{\omega}_{i b z}^{2}+\dot{\gamma}\right)=0 .
\end{gathered}
$$

When inner gimbal and outer gimbal are still on the ground, the dynamic equation of magnetically suspended rotor can be simplified as

$$
\begin{aligned}
& J_{r r} \ddot{\alpha}+H_{r z} \dot{\beta}=p_{x}, \\
& J_{r r} \ddot{\beta}-H_{r z} \dot{\alpha}=p_{y} .
\end{aligned}
$$

3.2. The Dynamic Model of Inner Gimbal. The absolute angular velocity $\overrightarrow{\boldsymbol{\omega}}_{i g}$ of inner gimbal is $\overrightarrow{\boldsymbol{\omega}}_{i g}=\overrightarrow{\boldsymbol{\omega}}_{i b}+\overrightarrow{\boldsymbol{\omega}}_{b s}+$ $\overrightarrow{\boldsymbol{\omega}}_{s j}+\overrightarrow{\boldsymbol{\omega}}_{j g}$.

(1) Taking inner gimbal frame $O x_{g} y_{g} z_{g}$ as moveable frame, the angular velocity of outer gimbal relative to zero position is $\overrightarrow{\boldsymbol{\omega}}_{s j}$. Its projection in inner gimbal frame is

$$
\overrightarrow{\boldsymbol{\omega}}_{s j}^{g}=\overrightarrow{\boldsymbol{\omega}}_{j g}+\mathbf{C}_{j}^{g} \overrightarrow{\boldsymbol{\omega}}_{s j}=\left[\begin{array}{c}
\dot{\theta}_{g} \\
\dot{\theta}_{j} \cos \theta_{g} \\
\dot{\theta}_{j} \sin \theta_{g}
\end{array}\right] .
$$

The zero position frame is still relative to body frame, so $\overrightarrow{\boldsymbol{\omega}}_{b s}=0$. The spacecraft rotation speed $\overrightarrow{\boldsymbol{\omega}}_{i b}$ in inner gimbal frame is denoted by

$$
\begin{aligned}
\overrightarrow{\boldsymbol{\omega}}_{i b}^{g} & =\left[\begin{array}{c}
\omega_{i b x}^{g} \\
\omega_{i b y}^{g} \\
\omega_{i b z}^{g}
\end{array}\right]=\mathbf{C}_{j}^{g} \mathbf{C}_{s}^{j} \mathbf{C}_{b}^{s} \overrightarrow{\boldsymbol{\omega}}_{i b}^{b} \\
= & {\left[\begin{array}{c}
\cos \theta_{j} \omega_{i b y}^{b}-\sin \theta_{j} \omega_{i b x}^{b} \\
\sin \theta_{g} \sin \theta_{j} \omega_{i b y}^{b}+\cos \theta_{g} \omega_{i b z}^{b}+\sin \theta_{g} \cos \theta_{j} \omega_{i b x}^{b} \\
\cos \theta_{g} \sin \theta_{j} \omega_{i b y}^{b}-\sin \theta_{g} \omega_{i b z}^{b}+\cos \theta_{g} \cos \theta_{j} \omega_{i b x}^{b}
\end{array}\right] . }
\end{aligned}
$$


So the absolute velocity $\overrightarrow{\boldsymbol{\omega}}_{i g}$ and the time derivative $\dot{\overrightarrow{\boldsymbol{\omega}}}_{i g}$ of inner gimbal can be described in inner gimbal frame as

$$
\begin{aligned}
\overrightarrow{\boldsymbol{\omega}}_{i g}^{g}= & \mathbf{C}_{b}^{g} \overrightarrow{\boldsymbol{\omega}}_{i b}+\mathbf{C}_{j}^{g} \overrightarrow{\boldsymbol{\omega}}_{s j}+\overrightarrow{\boldsymbol{\omega}}_{j g}=\overrightarrow{\boldsymbol{\omega}}_{i b}^{g}+\overrightarrow{\boldsymbol{\omega}}_{s j}^{g}+\overrightarrow{\boldsymbol{\omega}}_{j g}^{g} \\
= & {\left[\begin{array}{c}
\omega_{i b x}^{g}+\dot{\theta}_{g} \\
\omega_{i b y}^{g}+\dot{\theta}_{j} \cos \theta_{g} \\
\omega_{i b z}^{g}-\dot{\theta}_{j} \sin \theta_{g}
\end{array}\right] } \\
= & {\left[\begin{array}{c}
\cos \theta_{j} \omega_{i b y}^{b}-\sin \theta_{j} \omega_{i b x}^{b}+\dot{\theta}_{g} \\
\sin \theta_{g} \sin \theta_{j} \omega_{i b y}^{b}+\cos \theta_{g} \omega_{i b z}^{b} \\
+\sin \theta_{g} \cos \theta_{j} \omega_{i b x}^{b}+\dot{\theta}_{j} \cos \theta_{g} \\
\cos \theta_{g} \sin \theta_{j} \omega_{i b y}^{b}-\sin \theta_{g} \omega_{i b z}^{b} \\
+\cos \theta_{g} \cos \theta_{j} \omega_{i b x}^{b}-\dot{\theta}_{j} \sin \theta_{g}
\end{array}\right], } \\
& \dot{\vec{\omega}}_{i g}^{g}=\left[\begin{array}{c}
\dot{\omega}_{i b x}^{g}+\ddot{\theta}_{g} \\
\dot{\omega}_{i b y}^{g}+\ddot{\theta}_{j} \cos \theta_{g}-\dot{\theta}_{j} \dot{\theta}_{g} \sin \theta_{g} \\
\dot{\omega}_{i b z}^{g}-\ddot{\theta}_{j} \sin \theta_{g}-\dot{\theta}_{j} \dot{\theta}_{g} \cos \theta_{g}
\end{array}\right] .
\end{aligned}
$$

(2) The torque that acts on inner gimbal includes two parts, namely, the magnetic bearing torque and the outer gimbal torque. Then

$$
\mathbf{M}_{g r}^{1}=\left[\begin{array}{c}
-p_{x} \\
-p_{y} \\
0
\end{array}\right]
$$

The projection of this torque in inner gimbal frame is

$$
\mathbf{M}_{g r}^{g}=\mathbf{C}_{1}^{g} \mathbf{M}_{g r}^{1}=\left[\begin{array}{c}
-p_{x} \\
-p_{y} \\
0
\end{array}\right] .
$$
is

The torque acting on the inner gimbal by the outer gimbal

$$
\mathbf{M}_{g j}^{g}=\left[\begin{array}{c}
p_{g x} \\
p_{g y} \\
p_{g z}
\end{array}\right],
$$

where $p_{g y}$ and $p_{g z}$ are the constraint torque along $y_{g}$ and $z_{g}$ direction. $p_{g x}$ is along $x_{g}$ direction. So the total torque of inner gimbal resulting from the magnetic bearing and outer gimbal is

$$
\mathbf{M}_{g}^{g}=\mathbf{M}_{g r}^{g}+\mathbf{M}_{g j}^{g}=\left[\begin{array}{c}
p_{g x}-p_{x} \\
p_{g y}-p_{y} \\
p_{g z}
\end{array}\right] .
$$

(3) $n$ double-gimbal magnetically suspended CMGs are installed on spacecraft according to certain configurations, which can avoid singularity state. In inner gimbal frame
$O x_{g} y_{g} z_{g}$, the dynamic model of inner gimbal of the $i$ th $(i=$ $1, \ldots, n)$ double-gimbal magnetically suspended CMG is

$$
\overrightarrow{\boldsymbol{\omega}}_{i g}^{g} \times \mathbf{H}_{g i}+\dot{\mathbf{H}}_{g i}=\mathbf{M}_{g}^{g},
$$

where the angular momentum of inner gimbal of the $i$ th double-gimbal magnetically suspended CMG is $\mathbf{H}_{g i}=$ $\mathbf{J}_{g}\left(\mathbf{C}_{b}^{g} \overrightarrow{\boldsymbol{\omega}}_{i b}+\mathbf{C}_{j}^{g} \overrightarrow{\boldsymbol{\omega}}_{s j}+\overrightarrow{\boldsymbol{\omega}}_{j g}\right)$. Then

$$
\begin{aligned}
& \dot{\mathbf{H}}_{g i}=\mathbf{J}_{g}(\left(-\overrightarrow{\boldsymbol{\omega}}_{j g}^{\times} \mathbf{C}_{j}^{g} \mathbf{C}_{b}^{j}-\mathbf{C}_{j}^{g} \overrightarrow{\boldsymbol{\omega}}_{s j}^{\times} \mathbf{C}_{b}^{j}\right) \overrightarrow{\boldsymbol{\omega}}_{i b}+\mathbf{C}_{b}^{g} \dot{\overrightarrow{\boldsymbol{\omega}}}_{i b} \\
&\left.-\overrightarrow{\boldsymbol{\omega}}_{j g}^{\times} \mathbf{C}_{j}^{g} \overrightarrow{\boldsymbol{\omega}}_{s j}+\mathbf{C}_{j}^{g} \dot{\overrightarrow{\boldsymbol{\omega}}}_{s j}+\dot{\overrightarrow{\boldsymbol{\omega}}}_{j g}\right) .
\end{aligned}
$$
then

The moment of inertia of inner gimbal is $\left[\begin{array}{lll}J_{g x} & J_{g y} & J_{g z}\end{array}\right]^{T}$;

$$
\begin{aligned}
& J_{g x} \dot{\omega}_{i g x}^{g}+\left(J_{g z}-J_{g y}\right) \omega_{i g y}^{g} \omega_{i g z}^{g}=M_{g x}^{g}, \\
& J_{g y} \dot{\omega}_{i g y}^{g}+\left(J_{g x}-J_{g z}\right) \omega_{i g z}^{g} \omega_{i g x}^{g}=M_{g y}^{g}, \\
& J_{g z} \dot{\omega}_{i g z}^{g}+\left(J_{g y}-J_{g x}\right) \omega_{i g x}^{g} \omega_{i g y}^{g}=M_{g z}^{g} .
\end{aligned}
$$

Substituting (20), (21), and (25) into the above equation, the dynamic model of inner gimbal is

$$
\begin{aligned}
& J_{g x}\left(\dot{\omega}_{i b x}^{g}+\ddot{\theta}_{g}\right)+\left(J_{g z}-J_{g y}\right)\left(\omega_{i b y}^{g}+\dot{\theta}_{j} \cos \theta_{g}\right) \\
& \quad \cdot\left(\omega_{i b z}^{g}-\dot{\theta}_{j} \sin \theta_{g}\right)=p_{g x}-p_{x}, \\
& J_{g y}\left(\dot{\omega}_{i b y}^{g}+\ddot{\theta}_{j} \cos \theta_{g}-\dot{\theta}_{j} \dot{\theta}_{g} \sin \theta_{g}\right) \\
& \quad+\left(J_{g x}-J_{g z}\right)\left(\omega_{i b z}^{g}-\dot{\theta}_{j} \sin \theta_{g}\right)\left(\omega_{i b x}^{g}+\dot{\theta}_{g}\right)=p_{g y}-p_{y}, \\
& J_{g z}\left(\dot{\omega}_{i b z}^{g}-\ddot{\theta}_{j} \sin \theta_{g}-\dot{\theta}_{j} \dot{\theta}_{g} \cos \theta_{g}\right) \\
& \quad+\left(J_{g y}-J_{g x}\right)\left(\omega_{i b x}^{g}+\dot{\theta}_{g}\right)\left(\omega_{i b y}^{g}+\dot{\theta}_{j} \cos \theta_{g}\right)=p_{g z} .
\end{aligned}
$$

3.3. The Dynamic Model of Outer Gimbal. Supposing that $\overrightarrow{\boldsymbol{\omega}}_{s j}$ is the angular velocity of outer gimbal relative to zero position frame, $\overrightarrow{\boldsymbol{\omega}}_{b s}$ is the angular velocity of zero position frame relative to spacecraft body frame, and $\overrightarrow{\boldsymbol{\omega}}_{i b}$ is the implicated angular velocity of spacecraft body frame relative to inertial frame. The absolute angular velocity $\overrightarrow{\boldsymbol{\omega}}_{i j}$ of outer gimbal is $\overrightarrow{\boldsymbol{\omega}}_{i j}=\overrightarrow{\boldsymbol{\omega}}_{i b}+\overrightarrow{\boldsymbol{\omega}}_{b s}+\overrightarrow{\boldsymbol{\omega}}_{s j}$.

(1) Taking outer gimbal frame $O x_{j} y_{j} z_{j}$ as movable frame, zero position frame is kept stable relative to body frame: $\overrightarrow{\boldsymbol{\omega}}_{b s}=0$.

The spacecraft rotating velocity in inner gimbal frame is described as

$$
\overrightarrow{\boldsymbol{\omega}}_{i b}^{j}=\left[\begin{array}{c}
\omega_{i b x}^{j} \\
\omega_{i b y}^{j} \\
\omega_{i b z}^{j}
\end{array}\right]=\mathbf{C}_{s}^{j} \mathbf{C}_{b}^{s} \overrightarrow{\boldsymbol{\omega}}_{i b}^{b}=\left[\begin{array}{c}
\cos \theta_{j} \omega_{i b y}^{b}-\sin \theta_{j} \omega_{i b x}^{b} \\
\omega_{i b z}^{b} \\
\sin \theta_{j} \omega_{i b y}^{b}+\cos \theta_{j} \omega_{i b x}^{b}
\end{array}\right] .
$$


So the absolute angular velocity $\overrightarrow{\boldsymbol{\omega}}_{i j}^{j}$ of outer gimbal and its time derivative $\dot{\overrightarrow{\boldsymbol{\omega}}}_{i j}^{j}$ can be described as

$$
\begin{aligned}
\overrightarrow{\boldsymbol{\omega}}_{i j}^{j} & =\mathbf{C}_{b}^{j} \overrightarrow{\boldsymbol{\omega}}_{i b}+\overrightarrow{\boldsymbol{\omega}}_{s j} \\
& =\left[\begin{array}{c}
\omega_{i b x}^{j} \\
\omega_{i b y}^{j}+\dot{\theta}_{j} \\
\omega_{i b z}^{j}
\end{array}\right] \\
& =\left[\begin{array}{c}
\cos \theta_{j} \omega_{i b y}^{b}-\sin \theta_{j} \omega_{i b x}^{b} \\
\omega_{i b z}^{b}+\dot{\theta}_{j} \\
\sin \theta_{j} \omega_{i b y}^{b}+\cos \theta_{j} \omega_{i b x}^{b}
\end{array}\right], \\
\dot{\overrightarrow{\boldsymbol{\omega}}}_{i j}^{j} & =\left[\begin{array}{c}
-\sin \theta_{j} \dot{\theta}_{j} \omega_{i b y}^{b}+\cos \theta_{j} \dot{\omega}_{i b y}^{b}-\cos \theta_{j} \dot{\theta}_{j} \omega_{i b x}^{b} \\
-\sin \theta_{j} \dot{\omega}_{i b x}^{b} \\
\dot{\omega}_{i b z}^{b}+\ddot{\theta}_{j} \\
\cos \theta_{j} \dot{\theta}_{j} \omega_{i b y}^{b}+\sin \theta_{j} \dot{\omega}_{i b y}^{b}-\sin \theta_{j} \dot{\theta}_{j} \omega_{i b x}^{b} \\
+\cos \theta_{j} \dot{\omega}_{i b x}^{b} \\
\dot{\omega}_{i b x}^{j} \\
\dot{\omega}_{i b y}^{j}+\ddot{\theta}_{j} \\
\dot{\omega}_{i b z}^{j}
\end{array}\right] .
\end{aligned}
$$

(2) Similar with inner gimbal, the torque acting on outer gimbal is the torque from spacecraft and the torque from inner gimbal. The projection of inner gimbal torque on outer gimbal is

$$
\mathbf{M}_{j g}^{j}=\mathbf{C}_{g}^{j} \mathbf{M}_{j g}^{g}=\left[\begin{array}{c}
-p_{g x} \\
-p_{g y} \cos \theta_{j}-p_{g z} \sin \theta_{j} \\
p_{g y} \sin \theta_{j}-p_{g z} \cos \theta_{j}
\end{array}\right] .
$$

The torque that the spacecraft is acting on outer gimbal is

$$
\mathbf{M}_{j i}^{j}=\left[\begin{array}{c}
p_{j x} \\
p_{j y} \\
p_{j z}
\end{array}\right],
$$

where $p_{j y}$ is the outer gimbal motor driving torque along $y_{j}$ direction. $p_{j x}$ and $p_{j z}$ are the constraint torque in which the base acts on outer gimbal along $x_{j}$ and $z_{j}$ direction. So the total torque acting on outer gimbal is

$$
\mathbf{M}_{j}^{j}=\mathbf{M}_{j g}^{j}+\mathbf{M}_{j i}^{j}=\left[\begin{array}{c}
-p_{g x}+p_{j x} \\
-p_{g y} \cos \theta_{g}+p_{g z} \sin \theta_{g}+p_{j y} \\
-p_{g y} \sin \theta_{g}-p_{g z} \cos \theta_{g}+p_{j z}
\end{array}\right] .
$$

(3) In outer gimbal frame $O x_{j} y_{j} z_{j}$, the outer gimbal dynamic model of the $i$ th double-gimbal magnetically suspended CMG is

$$
\overrightarrow{\boldsymbol{\omega}}_{i j}^{j} \times \mathbf{H}_{j i}+\dot{\mathbf{H}}_{j i}=\mathbf{M}_{j}^{j},
$$

where the angular momentum of outer gimbal of the $i$ th double-gimbal magnetically suspended CMG is $\mathbf{H}_{j i}=$ $\mathbf{J}_{j}\left(\mathbf{C}_{b}^{j} \overrightarrow{\boldsymbol{\omega}}_{i b}+\overrightarrow{\boldsymbol{\omega}}_{s j}\right)$. Then $\dot{\mathbf{H}}_{j i}=\mathbf{J}_{j}\left(-\overrightarrow{\boldsymbol{\omega}}_{s j}^{\times} \mathbf{C}_{b}^{j} \overrightarrow{\boldsymbol{\omega}}_{i b}+\mathbf{C}_{b}^{j} \dot{\overrightarrow{\boldsymbol{\omega}}}_{i b}+\dot{\overrightarrow{\boldsymbol{\omega}}}_{s j}\right)$.

The moment of inertia of outer gimbal is $\left[\begin{array}{lll}J_{j x} & J_{j y} & J_{j z}\end{array}\right]^{T}$; then

$$
\begin{aligned}
& J_{j x} \dot{\omega}_{i j x}^{j}+\left(J_{j z}-J_{j y}\right) \omega_{i j y}^{j} \omega_{i j z}^{j}=M_{j x}^{j}, \\
& J_{j y} \dot{\omega}_{i j y}^{j}+\left(J_{j x}-J_{j z}\right) \omega_{i j z}^{j} \omega_{i j x}^{j}=M_{j y}^{j}, \\
& J_{j z} \dot{\omega}_{i j z}^{j}+\left(J_{j y}-J_{j x}\right) \omega_{i j x}^{j} \omega_{i j y}^{j}=M_{j z}^{j} .
\end{aligned}
$$

By substituting (31), (32), and (34) into the above equation, the dynamic equation of outer gimbal is

$$
\begin{gathered}
p_{j x}=J_{j x} \dot{\omega}_{i b x}^{j}+p_{g x}, \\
p_{j y}=J_{j y}\left(\dot{\omega}_{i b y}^{j}+\ddot{\theta}_{j}\right)+p_{g y} \cos \theta_{g}-p_{g z} \sin \theta_{g}, \\
p_{j z}=J_{j z} \dot{\omega}_{i b z}^{j}+p_{g y} \sin \theta_{g}+p_{g z} \cos \theta_{g} .
\end{gathered}
$$

\section{Agile Maneuver Spacecraft Dynamic Modelling Using Double-Gimbal Magnetically Suspended CMG as the Actuator}

The torque in spacecraft body frame can be described as

$$
\dot{\mathbf{H}}_{b}+\overrightarrow{\boldsymbol{\omega}}_{i b}^{b} \times \mathbf{H}_{b}=-\mathbf{M}_{b}^{b}+\mathbf{T}_{d}^{b}
$$

Supposing that the moment of inertia of the spacecraft is $\left[\begin{array}{lll}J_{b x} & J_{b y} & J_{b z}\end{array}\right]^{T}$, then

$$
\begin{aligned}
& J_{b x} \dot{\omega}_{i b x}^{b}+\left(J_{b z}-J_{b y}\right) \omega_{i b y}^{b} \omega_{i b z}^{b}=-M_{b x}^{b}+T_{d x}^{b}, \\
& J_{b y} \dot{\omega}_{i b y}^{b}+\left(J_{b x}-J_{b z}\right) \omega_{i b z}^{b} \omega_{i b x}^{b}=-M_{b y}^{b}+T_{d y}^{b}, \\
& J_{b z} \dot{\omega}_{i b z}^{b}+\left(J_{b y}-J_{b x}\right) \omega_{i b x}^{b} \omega_{i b y}^{b}=-M_{b z}^{b}+T_{d z}^{b},
\end{aligned}
$$

where $\mathbf{M}_{b}^{b}=\left[\begin{array}{lll}M_{b x}^{b} & M_{b y}^{b} & M_{b z}^{b}\end{array}\right]^{T}$ is the vector form of the total torque that acts on spacecraft. $\mathbf{T}_{d}^{b}=\left[\begin{array}{lll}T_{d x}^{b} & T_{d y}^{b} & T_{d z}^{b}\end{array}\right]^{T}$ is the disturbance, which can be described as

$$
\begin{aligned}
\mathbf{M}_{b}^{b}= & \sum_{i=1}^{n} \mathbf{M}_{j i}^{j} \\
= & \overrightarrow{\boldsymbol{\omega}}_{i b}^{\times} \sum_{i=1}^{n} \mathbf{C}_{r}^{b} \mathbf{h}_{0}+\sum_{i=1}^{n} \mathbf{C}_{j}^{b} \overrightarrow{\boldsymbol{\omega}}_{s j}^{\times} \mathbf{C}_{r}^{j} \mathbf{h}_{0}+\sum_{i=1}^{n} \mathbf{C}_{g}^{b} \overrightarrow{\boldsymbol{\omega}}_{j g}^{\times} \mathbf{C}_{r}^{g} \mathbf{h}_{0} \\
& +\sum_{i=1}^{n} \mathbf{C}_{r}^{b} \overrightarrow{\boldsymbol{\omega}}_{1 r}^{\times} \mathbf{h}_{0}+\sum_{i=1}^{n} \mathbf{T}_{j i}+\sum_{i=1}^{n} \mathbf{C}_{g}^{j} \mathbf{T}_{g i}+\sum_{i=1}^{n} \mathbf{T}_{r i},
\end{aligned}
$$


where $\mathbf{h}_{0}=\left[\begin{array}{lll}0 & 0 & \mathbf{I}_{r i} \gamma\end{array}\right]^{T}$ is the constant rotor angular momentum. The outer disturbance torque can be denoted by

$$
\begin{gathered}
\mathbf{T}_{g i}=\overrightarrow{\boldsymbol{\omega}}_{i g}^{g} \times \mathbf{H}_{g i}+\dot{\mathbf{H}}_{g i}, \\
\mathbf{T}_{j i}=\overrightarrow{\boldsymbol{\omega}}_{i j}^{j} \times \mathbf{H}_{j i}+\dot{\mathbf{H}}_{j i}, \\
\mathbf{T}_{r i}=\left\{\left(\overrightarrow{\boldsymbol{\omega}}_{i b}^{\times} \mathbf{C}_{r}^{b}+\mathbf{C}_{j}^{b} \overrightarrow{\boldsymbol{\omega}}_{s j}^{\times} \mathbf{C}_{r}^{j}+\mathbf{C}_{g}^{b} \overrightarrow{\boldsymbol{\omega}}_{j g}^{\times} \mathbf{C}_{r}^{g}+\mathbf{C}_{r}^{b} \overrightarrow{\boldsymbol{\omega}}_{1 r}^{\times}\right)\right. \\
\left.\cdot \mathbf{J}_{r}\left(\mathbf{C}_{b}^{r} \overrightarrow{\boldsymbol{\omega}}_{i b}+\mathbf{C}_{j}^{r} \overrightarrow{\boldsymbol{\omega}}_{s j}+\mathbf{C}_{g}^{r} \overrightarrow{\boldsymbol{\omega}}_{j g}+\overrightarrow{\boldsymbol{\omega}}_{1 r}\right)\right\} \\
+\mathbf{C}_{r}^{b} \mathbf{J}_{r}\left\{\left(-\overrightarrow{\boldsymbol{\omega}}_{1 r}^{\times} \mathbf{C}_{b}^{r}-\mathbf{C}_{g}^{r} \overrightarrow{\boldsymbol{\omega}}_{j g}^{\times} \mathbf{C}_{b}^{g}-\mathbf{C}_{j}^{r} \overrightarrow{\boldsymbol{\omega}}_{s j}^{\times} \mathbf{C}_{b}^{j}\right) \overrightarrow{\boldsymbol{\omega}}_{i b}\right. \\
+\mathbf{C}_{b}^{r} \dot{\overrightarrow{\boldsymbol{\omega}}}_{i b}+\left(-\overrightarrow{\boldsymbol{\omega}}_{1 r}^{\times} \mathbf{C}_{j}^{r}-\mathbf{C}_{g}^{r} \overrightarrow{\boldsymbol{\omega}}_{j g}^{\times} \mathbf{C}_{j}^{g}\right) \overrightarrow{\boldsymbol{\omega}}_{s j}+\mathbf{C}_{j}^{r} \dot{\overrightarrow{\boldsymbol{\omega}}}_{s j} \\
\left.-\overrightarrow{\boldsymbol{\omega}}_{1 r}^{\times} \mathbf{C}_{g}^{r} \overrightarrow{\boldsymbol{\omega}}_{j g}+\mathbf{C}_{g}^{r} \dot{\overrightarrow{\boldsymbol{\omega}}}{ }_{j g}+\dot{\overrightarrow{\boldsymbol{\omega}}}_{1 r}\right\} .
\end{gathered}
$$

Because the rotor displacement relative to the gimbal is small, $\mathrm{C}_{r}^{g} \approx \mathrm{E}, \mathrm{C}_{r}^{b} \approx \mathrm{C}_{g}^{b}$. Equation (41) can be described as

$$
\mathbf{T}=\dot{\mathbf{h}}+\overrightarrow{\boldsymbol{\omega}}_{i b} \times \mathbf{h}+\mathbf{T}_{r}
$$

where the total angular momentum of double-gimbal magnetically suspended CMGs is $\mathbf{h}=\sum_{i=1}^{n} \mathbf{C}_{r}^{b} \mathbf{h}_{0}$. Then

$$
\begin{aligned}
\dot{\mathbf{h}}= & \sum_{i=1}^{n} \mathbf{C}_{j}^{b} \overrightarrow{\boldsymbol{\omega}}_{s j}^{\times} \mathbf{C}_{r}^{j} \mathbf{h}_{0}+\sum_{i=1}^{n} \mathbf{C}_{g}^{b} \overrightarrow{\boldsymbol{\omega}}_{j g}^{\times} \mathbf{C}_{r}^{g} \mathbf{h}_{0} \approx \sum_{i=1}^{n} \mathbf{C}_{j}^{b} \overrightarrow{\boldsymbol{\omega}}_{s j}^{\times} \mathbf{C}_{g}^{j} \mathbf{h}_{0} \\
& +\sum_{i=1}^{n} \mathbf{C}_{g}^{b} \overrightarrow{\boldsymbol{\omega}}_{j g}^{\times} \mathbf{h}_{0}=\mathbf{J}(\boldsymbol{\sigma}) \dot{\boldsymbol{\sigma}},
\end{aligned}
$$

where $\boldsymbol{\sigma}=\left[\begin{array}{llllll}\sigma_{g 1} & \cdots & \sigma_{g n} & \sigma_{j 1} & \cdots & \sigma_{j n}\end{array}\right]^{T}$ is the inner gimbal angle and the outer gimbal angle of $n$ double-gimbal magnetically suspended CMGs. $\sigma_{g i}$ and $\sigma_{j i}$ are the gimbal rate of the $i$ th double-gimbal magnetically suspended CMG. $\mathbf{J}(\boldsymbol{\sigma})=\partial \mathbf{h} / \partial \boldsymbol{\sigma}$ is the Jacobin matrix. The outer disturbance torque can be described as

$$
\mathbf{T}_{r}=\sum_{i=1}^{n} \mathbf{C}_{r}^{b} \overrightarrow{\boldsymbol{\omega}}_{1 r}^{\times} \mathbf{h}_{0}+\sum_{i=1}^{n} \mathbf{T}_{j i}+\sum_{i=1}^{n} \mathbf{C}_{g}^{j} \mathbf{T}_{g i}+\sum_{i=1}^{n} \mathbf{T}_{r i} .
$$

Equations (16), (29), (38), and (40) form the dynamic model of double-gimbal magnetically suspended CMG when the spacecraft maneuvers. It can be seen that the relative motion of any component will result in the relative motion of other components. The motion of all components is coupling, which makes the dynamic model of spacecraft with doublegimbal magnetically suspended CMG complex.

\section{The Coupling Characteristic in Double- Gimbal Magnetically Suspended CMG}

Equations (16), (29), and (38) form the dynamic model of double-gimbal magnetically suspended CMG when spacecraft maneuvers. By writing them together, then

$$
\begin{gathered}
m \ddot{x}_{r}=f_{x}, \\
m \ddot{y}_{r}=f_{y}, \\
m \ddot{z}_{r}=f_{z}, \\
J_{r r}\left(\dot{\omega}_{i b x}^{2}+\ddot{\alpha}^{2} \ddot{\theta}_{g}\right)+H_{r z}\left(\omega_{i b y}^{2}+\dot{\beta}+\dot{\theta}_{j} \cos \theta_{g}\right)=p_{x}, \\
J_{r r}\left(\dot{\omega}_{i b y}^{2}+\ddot{\beta}+\ddot{\theta}_{j} \cos \theta_{g}-\dot{\theta}_{j} \dot{\theta}_{g} \sin \theta_{g}\right) \\
-H_{r z}\left(\omega_{i b x}^{2}+\dot{\alpha}+\dot{\theta}_{g}\right)=p_{y}, \\
J_{g x}\left(\dot{\omega}_{i b x}^{g}+\ddot{\theta}_{g}\right)+J_{r r}\left(\dot{\omega}_{i b x}^{2}+\ddot{\theta}_{g}\right)+J_{r r} \ddot{\alpha} \\
+H_{r z}\left(\omega_{i b y}^{2}+\dot{\beta}+\dot{\theta}_{j} \cos \theta_{g}\right)=p_{g x}, \\
+\ddot{\theta}_{j y}\left(\dot{\omega}_{i b y}^{j}+\ddot{\theta}_{j}\right)+J_{g y}\left(\dot{\omega}_{i b y}^{g}+\ddot{\theta}_{j}\right)+J_{r r}\left(\dot{\omega}_{i b y}^{2}+\ddot{\theta}_{j}\right) \\
+J_{r r} \ddot{\beta} \cos \theta_{g}-H_{r z}\left(\omega_{i b x}^{2}+\dot{\alpha}+\dot{\theta}_{g}\right) \cos \theta_{g}=p_{j y}, \\
J_{g x}\left(\dot{\omega}_{i b x}^{g}+\ddot{\theta}_{g}\right)+\left(J_{g z}-J_{g y}\right)\left(\omega_{i b y}^{g}+\dot{\theta}_{j} \cos \theta_{g}\right) \\
\left(\omega_{i b z}^{g}-\dot{\theta}_{j} \sin \theta_{g}\right)=p_{g x}-p_{x}, \\
J_{g y}\left(\dot{\omega}_{i b y}^{g}+\ddot{\theta}_{j} \cos \theta_{g}-\dot{\theta}_{j} \dot{\theta}_{g} \sin \theta_{g}\right) \\
+\left(J_{g x}-J_{g z}\right)\left(\dot{\omega}_{i b z}^{g}-\dot{\theta}_{j} \sin \theta_{g}\right)\left(\omega_{i b x}^{g}+\ddot{\theta}_{j}\right)+\dot{\theta}_{g y} \cos \theta_{g}-p_{g z} \sin \theta_{g}, \\
p_{j z}=J_{j z} \dot{\omega}_{i b z}^{j}+p_{g y} \sin \theta_{g}+p_{g z} \cos \theta_{g} . \\
\left.\ddot{\theta}_{j} \sin \theta_{g}-\dot{\theta}_{j} \dot{\theta}_{g} \cos \theta_{g}\right)
\end{gathered}
$$

From the above equations, the following can be seen.

(1) Equation (46) is the nonlinear dynamic model of the relative motion between magnetically suspended rotor, inner gimbal, and outer gimbal of double-gimbal magnetically suspended CMG when spacecraft maneuvers. These models are built in inner loop frame, inner gimbal frame, and outer gimbal frame, respectively. The relative motion of the three components must be considered for analyzing the spacecraft stability and precision.

(2) The magnetic bearing suspending with gap is used in double-gimbal magnetically suspended CMG. The motion of magnetically suspended rotor does not only rely on magnetic 
bearing force but also suffer from the influence of gimbal servo system and spacecraft motion.

(3) Between the inner gimbal and the outer gimbal, there exists the dynamic coupling that results from the gyro effect. This dynamic coupling not only depends on the gimbal motion but also is related with the radial rotation. When spacecraft maneuvers, the gyro coupling torque of double-gimbal magnetically suspended CMG is proportional to spacecraft angular velocity and the cosine of the radial angle displacement. So this system is a nonlinear system. The disturbance influence of the strong coupling torque on the magnetically suspended rotor can make the magnetically suspended rotor unstable.

In (46), $p_{x}$ and $p_{y}$ can be obtained by using the dynamic equation of magnetically suspended rotor, namely, (16). $p_{g x}$, $p_{g y}$, and $p_{g z}$ can be obtained by substituting them into the dynamic model of inner gimbal, namely, (29). And then, they are substituted into the dynamic equation of outer gimbal, namely, (38), to obtain $p_{j x}, p_{j y}$, and $p_{j y}$. By ignoring the highorder item, the dynamic model of magnetic bearing along $x$ direction, $y$-direction of the inner and outer gimbal can be obtained:

$$
\begin{gathered}
J_{r r} \ddot{\alpha}+H_{r z} \dot{\beta}+J_{r r}\left(\dot{\omega}_{i b x}^{2}+\ddot{\theta}_{g}\right)+H_{r z}\left(\omega_{i b y}^{2}+\dot{\theta}_{j} \cos \theta_{g}\right)=p_{x}, \\
J_{r r} \ddot{\beta}-H_{r z} \dot{\alpha}+J_{r r}\left(\dot{\omega}_{i b y}^{2}+\ddot{\theta}_{j} \cos \theta_{g}\right)-H_{r z}\left(\omega_{i b x}^{2}+\dot{\theta}_{g}\right)=p_{y}, \\
J_{r r} \ddot{\alpha}+H_{r z} \dot{\beta}+J_{g x}\left(\dot{\omega}_{i b x}^{g}+\ddot{\theta}_{g}\right)+J_{r r}\left(\dot{\omega}_{i b x}^{2}+\ddot{\theta}_{g}\right) \\
\quad+H_{r z}\left(\omega_{i b y}^{2}+\dot{\theta}_{j} \cos \theta_{g}\right)=p_{g x}, \\
J_{r r} \ddot{\beta} \cos \theta_{g}-H_{r z}\left(\omega_{i b x}^{2}+\dot{\theta}_{g}+\dot{\alpha}\right) \cos \theta_{g}+J_{j y}\left(\dot{\omega}_{i b y}^{j}+\ddot{\theta}_{j}\right) \\
+J_{g y}\left(\dot{\omega}_{i b y}^{g}+\ddot{\theta}_{j}\right)+J_{r r}\left(\dot{\omega}_{i b y}^{2}+\ddot{\theta}_{j}\right)=p_{j y} .
\end{gathered}
$$

Based on the dynamic model of double-gimbal magnetically suspended CMG in (46), the coupling torque along $x$ direction of magnetic bearing, $y$-direction of magnetic bearing, the inner gimbal, and the outer gimbal can be obtained. The coupling torque can be divided into two portions, including the gyro coupling torque and the inertial coupling torque, where the gyro coupling torque is proportional to the spacecraft angular velocity. The gyro coupling torque is

$$
\begin{gathered}
M_{g c 2 x}=H_{r z}\left(\omega_{i b y}^{2}+\dot{\theta}_{j} \cos \theta_{g}\right), \\
M_{g c 2 y}=-H_{r z}\left(\omega_{i b x}^{2}+\dot{\theta}_{g}\right), \\
M_{g c g x}=H_{r z}\left(\omega_{i b y}^{2}+\dot{\theta}_{j} \cos \theta_{g}\right), \\
M_{g c j y}=-H_{r z}\left(\omega_{i b x}^{2}+\dot{\theta}_{g}\right) \cos \theta_{g},
\end{gathered}
$$

where $M_{g c 2 x}, M_{g c 2 y}, M_{g c g x}$, and $M_{g c j y}$ are the gyro coupling torque along $x$-direction of magnetic bearing, $y$-direction of magnetic bearing, the inner gimbal, and the outer gimbal, respectively. The inertial coupling torque is proportional to the angle acceleration of the spacecraft and gimbal:

$$
\begin{gathered}
M_{i c 2 x}=J_{r r}\left(\dot{\omega}_{i b x}^{2}+\ddot{\theta}_{g}\right), \\
M_{i c 2 y}=J_{r r}\left(\dot{\omega}_{i b y}^{2}+\ddot{\theta}_{j} \cos \theta_{g}\right), \\
M_{i c g x}=J_{g x}\left(\dot{\omega}_{i b x}^{g}+\ddot{\theta}_{g}\right)+J_{r r}\left(\dot{\omega}_{i b x}^{2}+\ddot{\theta}_{g}\right), \\
M_{i c j y}=J_{j y}\left(\dot{\omega}_{i b y}^{j}+\ddot{\theta}_{j}\right)+J_{g y}\left(\dot{\omega}_{i b y}^{g}+\ddot{\theta}_{j}\right)+J_{r r}\left(\dot{\omega}_{i b y}^{2}+\ddot{\theta}_{j}\right),
\end{gathered}
$$

where $M_{i c 2 x}, M_{i c 2 y}, M_{i c g x}$, and $M_{i c j y}$ are the inertial coupling torque along $x$-direction of magnetic bearing, $y$-direction of magnetic bearing, the inner gimbal, and the outer gimbal, respectively.

\section{Conclusion}

Double-gimbal magnetically suspended CMG is a novel attitude control actuator for the agile maneuver spacecraft. The rotor has five degrees of freedom besides the rotation degree, including three translation motions and two radial motions. So the dynamic model of the double-gimbal magnetically suspended CMG is complex. Moreover, the maneuver of spacecraft will influence the stability and precision of high speed rotor.

In order to verify the interrelation in double-gimbal magnetically suspended CMG, its working principal and basic structure are given firstly. Then, the dynamic model of the magnetically suspended rotor, inter gimbal, outer gimbal, and spacecraft using double-gimbal magnetically suspended control moment gyro as actuator is built. From these models, it can be seen that the model of one component includes the coupling moment produced by other components, and the relative motion of one component will affect the motion of other components. The strong coupling between the components is investigated, which will provide the foundation for the further research.

\section{Nomenclature}

$f_{x}$ : Radial force in $x$ direction

$f_{y}$ : Radial force in $y$ direction

$p_{x}$ : The radial torque in $x$ direction

$p_{y}$ : The radial torque in $y$ direction

$\mathbf{J}_{b}$ : The moments of inertia of spacecraft

$\mathbf{J}_{j}$ : The moments of inertia of outer gimbal

$\mathbf{J}_{g}$ : The moments of inertia of inner gimbal

$\mathbf{J}_{r}$ : The moments of inertia of rotor

$\alpha$ : The rotation of rotor frame relative to magnetic bearing stator rotating about the inner loop rotation axis

$\beta$ : The rotation of rotor frame relative to magnetic bearing stator rotating about outer loop rotation axis

$\gamma$ : The rotation of rotor frame relative to magnetic bearing stator rotating about the rotor spin axis 
$\theta_{g}:$ The rotation of the magnetic bearing stator frame relative to inner gimbal frame

$\theta_{j}$ : The rotation of outer gimbal frame relative to inertial frame

$\overrightarrow{\boldsymbol{\omega}}_{i r}$ : The angle speed vector of magnetically suspended rotor

$\overrightarrow{\boldsymbol{\omega}}_{1 r}$ : The rotation speed of rotor frame relative to stator frame

$\overrightarrow{\boldsymbol{\omega}}_{g 1}$ : The rotation speed of stator frame relative to inner gimbal frame

$\overrightarrow{\boldsymbol{\omega}}_{j g}$ : The rotation speed of inner gimbal frame relative to outer gimbal frame

$\overrightarrow{\boldsymbol{\omega}}_{s j}$ : The rotation speed of outer gimbal frame relative to zero position frame

$\vec{\omega}_{b s}$ : The rotation speed of zero position frame relative to body frame

$\overrightarrow{\boldsymbol{\omega}}_{i b}$ : The rotation speed of body frame relative to inertial frame.

\section{Conflict of Interests}

The authors declare that there is no conflict of interests regarding the publication of this paper.

\section{Acknowledgments}

This research has been supported by National Natural Science Foundation of China under Grant 61121003, the National Basic Research Program (973 Program) of China under Grant 2009CB72400101C, and National Civil Aerospace PreResearch Project.

\section{References}

[1] J. C. Fang, X. B. Xu, J. Q. Tang, and H. Liu, "Adaptive complete suppression of imbalance vibration in AMB systems using gain phase modifier," Journal of Sound and Vibration, vol. 332, no. 24, pp. 6203-6215, 2013.

[2] J. C. Fang, X. B. Xu, and J. J. Xie, "Active vibration control of rotor imbalance in active magnetic bearing systems," Journal of Vibration and Control, vol. 21, no. 4, pp. 684-700, 2015.

[3] R. J. Bauer, "Kinematics and dynamics of a double-gimbaled control moment gyroscope," Mechanism and Machine Theory, vol. 37, no. 12, pp. 1513-1529, 2002.

[4] B. Wie, "Singularity escape/avoidance steering logic for control moment gyro systems," Journal of Guidance, Control, and Dynamics, vol. 28, no. 5, pp. 948-956, 2005.

[5] J. C. Fang and Y. Ren, "Decoupling control of magnetically suspended rotor system in control moment gyros based on an inverse system method," IEEE/ASME Transactions on Mechatronics, vol. 17, no. 6, pp. 1133-1144, 2012.

[6] J. Fang and Y. Ren, "Self-adaptive phase-lead compensation based on unsymmetrical current sampling resistance network for magnetic bearing switching power amplifiers," IEEE Transactions on Industrial Electronics, vol. 59, no. 2, pp. 1218-1227, 2012.

[7] J. Fang, S. Zheng, and B. Han, "AMB vibration control for structural resonance of double-gimbal control moment gyro with high-speed magnetically suspended rotor," IEEE/ASME Transactions on Mechatronics, vol. 18, no. 1, pp. 32-43, 2013.

[8] C. J. Heiberg, "A practical approach to modeling SingleGimbal control momentum gyroscopes in agile spacecraft," in Proceedings of the Guidance, Navigation, and Control Conference and Exhibit, Denver, Colo, USA, August 2000.

[9] J. Ahmed and D. S. Bernstein, "Adaptive control of doublegimbal control-moment gyro with unbalanced rotor," Journal of Guidance, Control, and Dynamics, vol. 25, no. 1, pp. 105-115, 2002.

[10] L. Tang and Y. Chen, "Modeling and dynamic analysis of double-gimbal control moment gyros," Acta Aeronautica et Astronautica Sinica, vol. 29, no. 2, pp. 424-429, 2008.

[11] H.-D. Duan and Z. Wu, "Dynamics and simulation of spacecraft with magnetically suspended control moment gyroscope," Journal of System Simulation, vol. 21, no. 9, pp. 2783-2790, 2009 (Chinese).

[12] Y. Ren and J. Fang, "High-stability and fast-response twisting motion control for the magnetically suspended rotor system in a control moment gyro," IEEE/ASME Transactions on Mechatronics, vol. 18, no. 5, pp. 1625-1634, 2013.

[13] M. Xiang and T. Wei, "Autobalancing of high-speed rotors suspended by magnetic bearings using LMS adaptive feedforward compensation," Journal of Vibration and Control, vol. 20, no. 9, pp. 1428-1436, 2014.

[14] H.-T. Li, J.-C. Fang, B.-C. Han, and T. Wei, "Study on the system disturbance rejection method used in the gimbal servo system of double gimbal magnetically suspended control moment gyro," Journal of Astronautics, vol. 30, no. 6, pp. 2199-2205, 2009 (Chinese). 


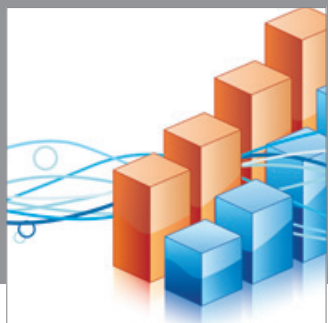

Advances in

Operations Research

mansans

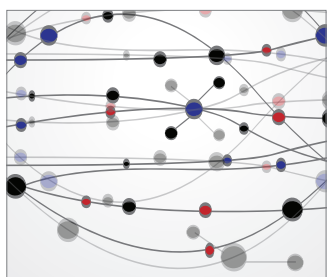

The Scientific World Journal
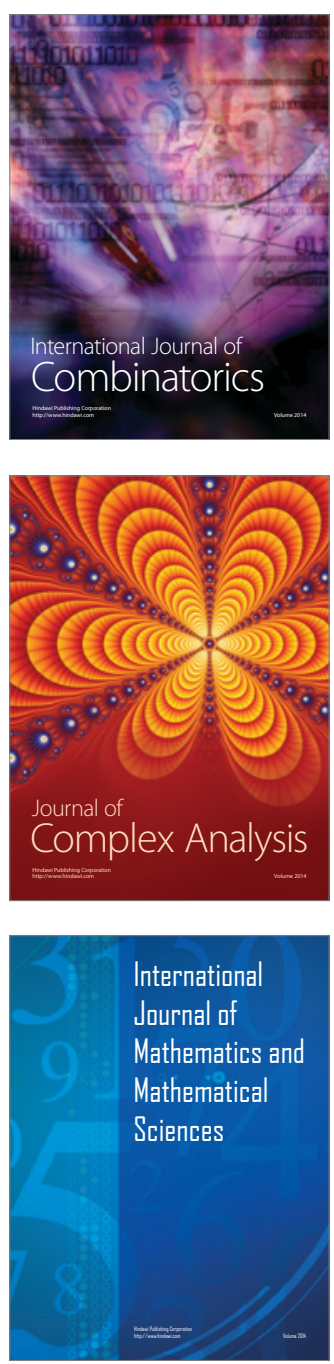
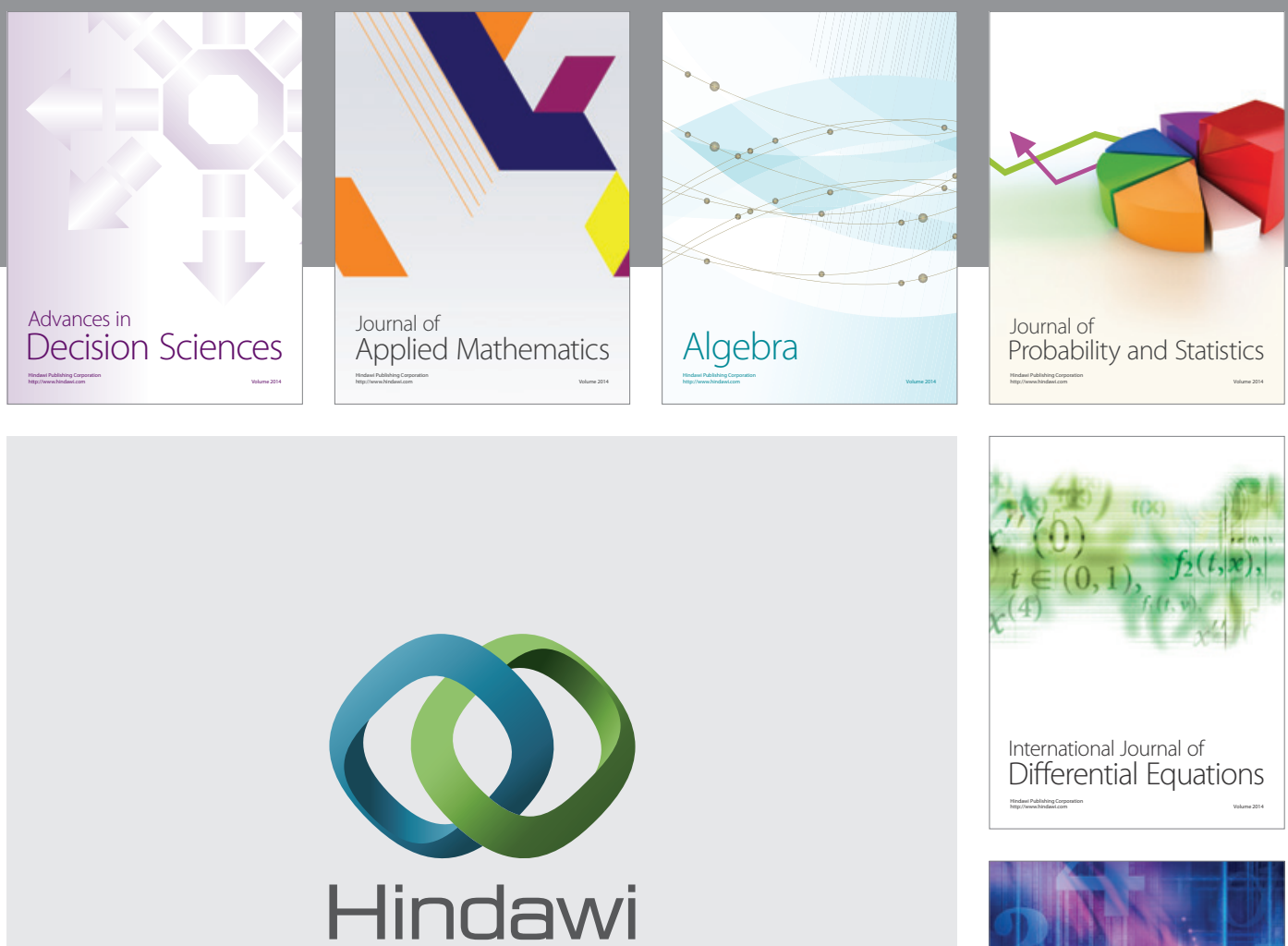

Submit your manuscripts at http://www.hindawi.com
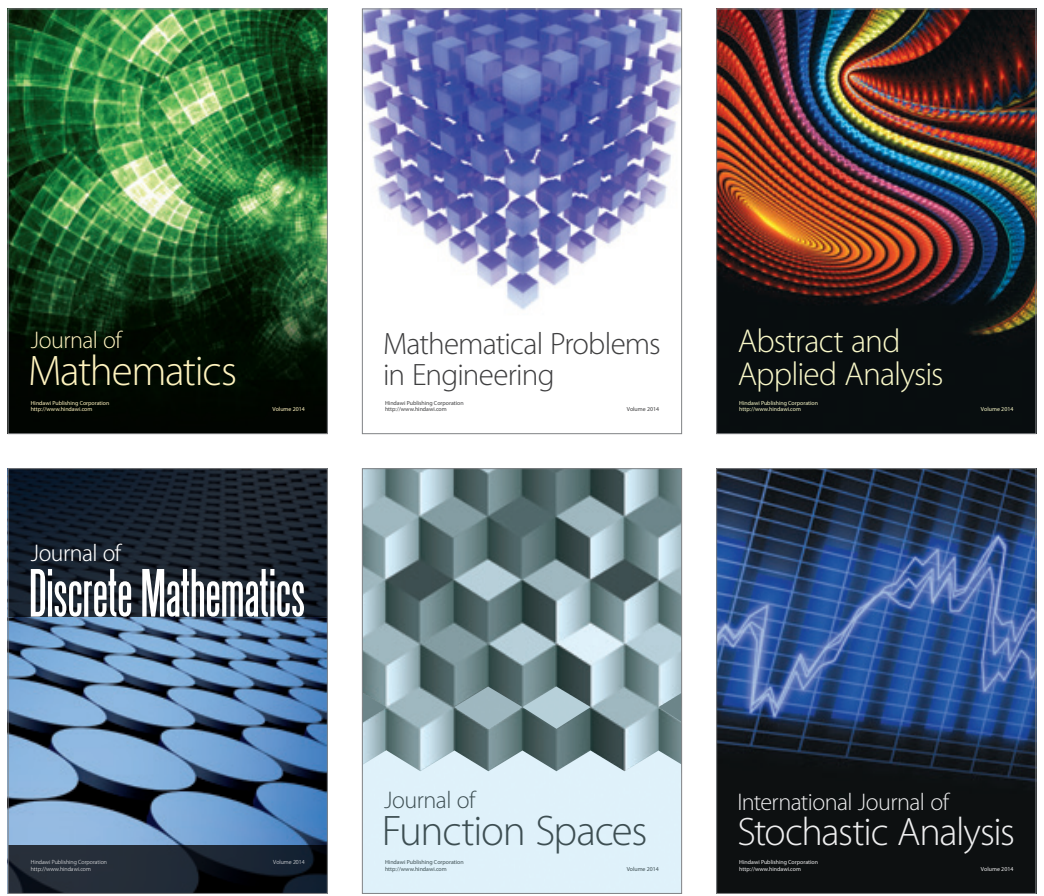

Journal of

Function Spaces

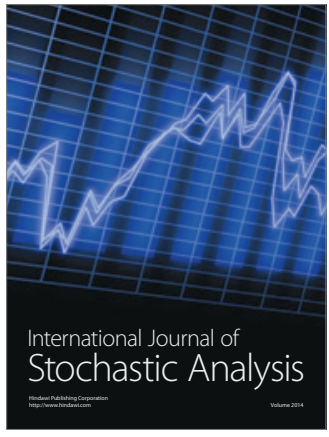

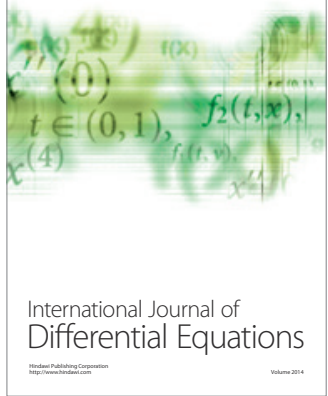
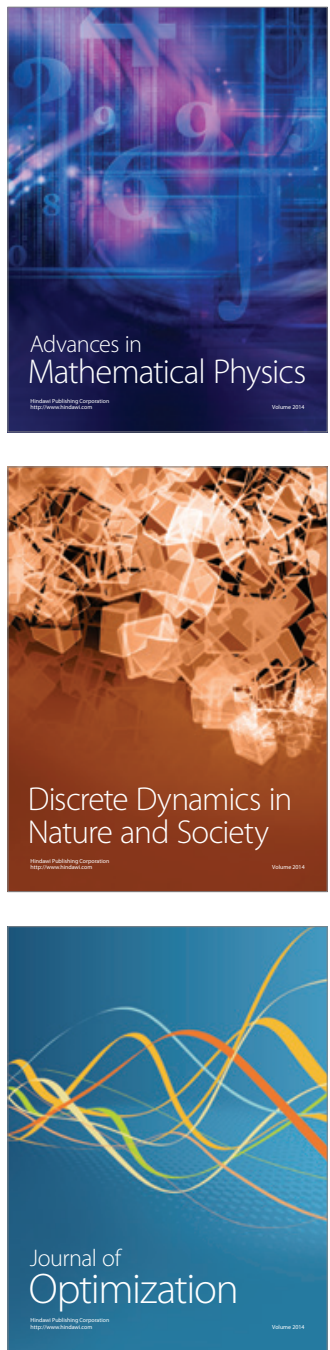